\title{
PAHs and star formation in ELAIS N1 as seen by AKARI
}

\author{
Tímea Kovács ${ }^{1} \mathbb{D}$, Denis Burgarella ${ }^{2}$, Hidehiro Kaneda ${ }^{3}$, \\ Dániel Cs. Molnár ${ }^{4}$, Shinki Oyabu ${ }^{3}$, Sandor Pinter ${ }^{1}{ }^{\circledR}$ and \\ L. Viktor $\operatorname{Toth}^{1,5}$ (i) \\ ${ }^{1}$ Department of Astronomy of the Eötvös Loránd University, Pázmány Péter sétány 1/A, \\ H-1117 Budapest, Hungary \\ ${ }^{2}$ Aix-Marseille Université, CNRS, LAM (Laboratoire d'Astrophysique de Marseille), UMR \\ 7326, 13388 Marseille, France \\ ${ }^{3}$ Graduate School of Science, Nagoya University, Japan \\ ${ }^{4}$ INAF - Osservatorio Astronomico di Cagliari, Via della Scienza 5, I-09047 Selargius (CA), \\ Italy \\ ${ }^{5}$ Konkoly Observatory of the Hungarian Academy of Sciences, Konkoly Thege Miklós út \\ 15-17., H-1121 Budapest, Hungary
}

\begin{abstract}
We have examined the relationship between star formation and polycyclic aromatic hydrocarbons (PAHs) by fitting the spectral energy distributions (SED) of AKARI selected galaxies. PAHs are excited by the ultraviolet (UV) photons of young stars and can trace star formation in galaxies, but they are disassociated by the strong UV radiation in starbursts. AKARI covered the mid-infrared, where the PAHs emit their radiation, with a high density of photometric bands. These observations allow us to estimate the star formation rate and the PAH mass fraction of the dust in galaxies. In the future the James Webb Space Telescope (JWST) will also make measurements in this wavelength range. This research can therefore be considered as a pathfinder to similar studies that will come later from JWST observations.
\end{abstract}

Keywords. galaxies: ISM, galaxies: star formation, infrared: galaxies

\section{Data and Methods}

We selected sources with Herschel and AKARI detections, and known redshifts in the ELAIS (European Large Area ISO Survey) N1 field (which was observed from the optical to the radio). We used photometric data from the Herschel Point Source Catalogs (Marton et al. 2017; Schulz et al. 2017) to constrain the far-infrared radiation, and from the AKARI Deep Field Catalogs (Davidge et al. 2017, 4 - 18 microns) to estimate the $\mathrm{PAH}$ emission. Photometric data at other wavelengths was also collected from different databases (for example WISE, SDSS).

We fitted the spectral energy distributions (SED) of galaxies with CIGALE (Noll et al. 2009; Roehlly et al. 2014; Boquien et al. 2019), and derived their physical properties like stellar mass, star formation rate (SFR), dust mass and the mass fraction of PAH molecules $\left(q_{\mathrm{PAH}}\right)$. We used the Bruzual \& Charlot $(2003)$ stellar emission models with a Chabrier (2003) initial mass function, a delayed star formation history, the Draine \& Li (2007) dust emission model, and a double powerlaw for the attenuation.

\section{Results}

Using the AKARI photometric data in the SED fitting lowered the error of the $q_{\mathrm{PAH}}$ parameter on average: the mean relative error of $q_{\mathrm{PAH}}$ dropped from $37 \%$ to $26 \%$. The 
models often under or overestimated the PAH emission without the AKARI data. When using AKARI points in the SEDs the $q_{\mathrm{PAH}}$ parameter became significantly lower in some cases, and higher in others.

The $q_{\mathrm{PAH}}$ in the selected sample is lower than the values usually found in previous works. This could be due to various reasons, such as low metallicity, ongoing active galactic nucleus or starburst activity. The spectrum of one of our sources has line ratios characteristic of AGNs, and another one has low metallicity. Based on the results of the SED fitting, the majority of the sample is on the main sequence of galaxies (Schreiber et al. 2015), only $8 \%$ of them could be starbursts. We found a decreasing $q_{\mathrm{PAH}}$ trend with increasing $A_{\mathrm{V}}$ and consequently LIR, suggesting the possible presence of compact star-forming regions.

We investigated different known relations, for example the PAH luminosities were calculated from the fitted SEDs, and compared to the SFR derived by CIGALE: the two methods give a similar SFR (Shipley et al. 2016).

\section{Discussion and summary}

To find the reason behind the low $q_{\mathrm{PAH}}$ values (e. g. determining the metallicity) follow-up spectroscopy would be needed.

The James Webb Space Telescope will have spectroscopic and photometric instruments which are sensitive in the near and mid-infrared $(0.6-28.3$ micron $)$. It will be able to measure the equivalent widths of the 3.3 and the $7.7 \mu \mathrm{m}$ PAH features up to $\mathrm{z} \sim 6$ and $\mathrm{z} \sim 2.5$, respectively. Similarly to the AKARI filters, the MIRI instrument will have 9 broadband filters at wavelengths from 4.6 to $28.8 \mu \mathrm{m}$ (Gardner et al. 2006). The results based on the AKARI selected galaxies are summarized in a paper: Kovács et al. (2019).

\section{Acknowledgements}

This research was supported by the Hungarian OTKA grant NN111016, and by the ÚNKP-18-2 New National Excellence Program of the Ministry of Human Capacities. This research has made use of the SIMBAD database, and the VizieR catalog access tool, CDS, Strasbourg, France. We made use of data products from the Wide-field Infrared Survey Explorer, and the research is based on observations with AKARI, a JAXA project with the participation of ESA. The conference participation was supported by the IAU.

\section{References}

Boquien, M., Burgarella, D., Roehlly, Y., et al. 2019, A $₫ A$, 622, A103

Bruzual, G. \& Charlot, S. 2003, MNRAS, 344, 1000

Chabrier, G. 2003, PASP, 115, 763

Davidge, H., Serjeant, S., Pearson, C., et al. 2017, MNRAS, 472, 4259

Draine, B. T. \& Li, A. 2007, ApJ, 657, 810

Gardner, J. P., Mather, J. C., Clampin, M., et al. 2006, SSR, 123, 485

Kovács, T. O., Burgarella, D., Kaneda, H., et al. 2019, PASJ, 71, 27

Marton, G., Calzoletti, L., Perez Garcia, A. M., et al. 2017, arXiv:1705.05693

Noll, S., Burgarella, D., Giovannoli, E., et al. 2009, A\&GA, 507, 1793

Roehlly, Y., Burgarella, D., Buat, V., et al. 2014, Astronomical Data Analysis Software and Systems XXIII, 485, 347

Schulz, B., Marton, G., Valtchanov, I., et al. 2017, arXiv:1706.00448

Schreiber, C., Pannella, M., Elbaz, D., et al. 2015, A 6 A, 575, A74

Shipley, H. V., Papovich, C., Rieke, G. H., et al. 2016, ApJ, 818, 60 